\title{
Predator-induced life-history changes and the coexistence of five taxa in a Daphnia species complex
}

\author{
Piet Spaak, Joost Vanoverbeke and Maarten Boersma
}

Spaak, P., Vanoverbeke, J. and Boersma, M. 2000. Predator-induced life-history changes and the coexistence of five taxa in a Daphnia species complex. - Oikos 89: $164-174$.

\begin{abstract}
Interspecific hybridization is common in water fleas of the Daphnia galeata species complex (e.g. D. galeata, D. cucullata, D. hyalina and their interspecific hybrids). We studied the effect of fish on the life histories of five taxa of this species complex originating from the Plußsee, northern Germany. Using four clones per taxon, we found that fish kairomones reduce size at birth and size at maturity significantly. For size at maturity larger taxa showed a significantly stronger reaction to fish kairomones than the smaller taxa. With respect to the intrinsic rate of increase, $r$, we compared two predation regimes (positive size selective and not selective). We found that under the fish predation regime most clones had a higher $r$ when cultured with fish kairomones, leading to a higher $r$, and a stronger reaction for the smaller taxa. We conclude that fish predation might be an important factor influencing the co-occurrence of Daphnia parental taxa with their hybrids.
\end{abstract}

P. Spaak, Dept of Limnology, EAWAG/ETH, Überlandstrasse 133, CH-8600 Dübendorf, Switzerland (spaak@eawag.ch). - J. Vanoverbeke, Laboratory of Aquatic Ecology, Catholic Univ. of Leuven, De Beriotstraat 32, B-3000 Leuven, Belgium. - M. Boersma, Max-Planck-Institut für Limnologie, Postfach 165, D-24302 Plön, Germany.

Hybridization, the mating of genetically distinct groups of individuals, resulting in at least some offspring of mixed ancestry (Harrison 1993, Arnold 1997) has been of great interest to ecologists and evolutionary biologists for over a century (Darwin 1859, Mayr 1942, 1963, Grant and Grant 1992). In contrast to relatively slow evolutionary processes like genetic drift and natural selection, hybridization is a dramatic process that creates individuals with completely novel combinations of genes within one generation.

The evolutionary role of hybridization processes has been the subject of considerable debate. Traditionally, hybrids were considered as evolutionary dead ends, as the more conspicuous (mammalian) interspecific hybrids are mostly sterile. However, recent investigations on hybrids in the plant kingdom suggest that hybridization events have in fact resulted in the origin of many taxa. Stace (1987) for example, has argued that 50 to $70 \%$ of extant angiosperms originate from hybridiza- tion events (see also Grant 1981, Whitham et al. 1991). Also in the animal kingdom, hybridization is probably a much more frequent process than previously believed (Solignac and Monnerot 1986, Grant 1993).

Two processes play a role in the determination of hybrid abundance. First, the frequency of the production of hybrids by the parental taxa in a hybrid zone is of importance, but obviously also their relative fitness and persistence determine hybrid success. Most theories on the maintenance of hybrid zones are based on the observation that hybrid fitness is typically lower (Barton and Hewitt 1985, 1989). According to these theories two taxa meet and hybridize in a 'tension zone', but due to the lower fitness of the hybrids recurrent hybridization events are needed to keep the zone intact. Through these processes, the zone remains relatively narrow. Another class of models is based on the assumption that the relative fitness of hybrids and parental genotypes is largely determined by ecological

Accepted 25 August 1999

Copyright C OIKOS 2000

ISSN 0030-1299

Printed in Ireland - all rights reserved 
factors (e.g. hybrid superiority model, gradient and ecotone models; Endler 1977, Moore 1977). Under the conditions described by these models, the parental taxa remain genetically distinct, but the co-occurrence with their interspecific hybrids allows the transfer of genes from one taxon to the other. This process increases the number of genotypes and as a consequence the abilities of the parental species to react to changing environmental conditions (Anderson and Stebbins 1954).

Over the past two decades, several hybrid species complexes have been described in ecologically important taxa of freshwater zooplankton (Hann and Hebert 1982, Shan and Frey 1983, Hebert and Payne 1985, Wolf and Mort 1986, Lieder 1987). In particular, species of the genus Daphnia (Crustacea, Anomopoda) have been studied extensively (Schwenk and Spaak 1995). Within the Daphnia galeata complex (D. galeata Sars, D. cucullata Sars and D. hyalina Leydig) hybridization is quite common. These hybrids are found in many European and North American lakes, in which they are often the dominant taxa and often co-occur with one or both parental species (for review see Schwenk and Spaak 1997).

Within a growing season, Daphnia species reproduce parthenogenetically, and only when growing conditions deteriorate does sexual reproduction take place with the production of resting eggs. Although hybrids also seem capable of reproducing sexually (Schwenk 1997), it is still unclear as to how successful sexual offspring of hybrids really are. However, hybrids produce direct-developing offspring parthenogenetically, and hence during a growing season this mode of reproduction enables the hybrids to establish high population densities (e.g. Spaak and Hoekstra 1997). In deeper lakes, which do not completely freeze, hybrids are even known to survive the winter as parthenogenetic individuals (Weider and Stich 1992, Spaak 1996). In fact, detailed studies on life histories of hybrids have shown that as a result of the blend of parental traits, some environmental conditions might actually favour hybrids in comparison with the parental species (Boersma and Vijverberg 1994a, Spaak and Hoekstra 1995, 1997). Indeed, Schwenk (1997) observed correlations of certain lake characteristics with the frequency of hybrids in these lakes. Moreover, most lakes undergo dramatic changes within an annual cycle (e.g. Sommer et al. 1986). This implies that not only among lakes, but also within lakes, environmental circumstances may periodically be such that they favour hybrids.

In aquatic environments, predation is one of the most important factors influencing community structure and the evolution of life-history characteristics of zooplankton (e.g. Lynch 1980, Zaret 1980, Kerfoot and Sih 1987). Species of the $D$. galeata complex normally occur in lakes, and fish are the most important predators in these environments. Results from the life-history studies cited above indicate that for example in Tjeuke- meer, The Netherlands, a combination of high food levels with moderate predation levels would indeed favour the hybrids between $D$. galeata and $D$. cucullata over the parental species. This is a result of the combination of the relatively small size (avoiding positive-size selective predators) of $D$. cucullata, and high egg production in D. galeata.

Not only the intrinsic character traits of prey are of importance to an individual's success, but also how these traits vary in the presence of predators determines the survival of an individual. Daphnia is known to respond to the presence of fish by changing its morphology, life history and behaviour. Several studies (for review see Larsson and Dodson 1993) have indicated that the mere presence of chemicals released by predators (kairomones) can cause life-history shifts in cladocerans in the direction predicted by theoretical models (Taylor and Gabriel 1992, Fiksen 1997). It has been shown, for example, that in the presence of fish kairomones, size at maturity and the age at maturity decrease (Stibor 1992, Reede 1995, Reede and Ringelberg 1995), but the main shortcoming of the studies cited above is that these were performed with one taxon only, and maximally two clones. Furthermore, no studies on species of the genus Daphnia exist that have investigated the reactions of different coexisting taxa to the presence of fish kairomones.

We performed a life-history experiment with 20 clones isolated from the Plußsee in northern Germany, a lake in which $D$. galeata and $D$. cucullata coexist with the hybrid taxa $D$. cucullata $\times$ galeata, D. galeata $\times$ hyalina and D. cucullata $\times$ hyalina (Spaak 1995a). We hypothesise that the life-history reaction of the different taxa to the presence of chemicals exuded by fish is directly related to the mean size of the different taxa. Taxa with a higher mean size ('large taxa'), which are more vulnerable to predation by positively size-selective predators, are expected to show a stronger response than taxa with a lower mean size ('smaller taxa'). To test the effect of these different life histories on the survival chances under size-specific predation we used the model of Taylor and Gabriel (1992). We compared the effect of a positive size-selective predation scenario (fish) with a non-selective predation scenario.

\section{Material and methods}

\section{Origin of the clones}

All of the clones used in this experiment originated from the Plußsee (northern Germany). The Plußsee is a small (14 ha) funnel-shaped eutrophic lake surrounded by trees. It is stratified for most of the year, with an anoxic zone starting between 6 and $8 \mathrm{~m}$. Mean depth of the lake is $9.4 \mathrm{~m}$ and the maximum depth is $27 \mathrm{~m}$. The Daphnia community of this lake consists of D. galeata, 
D. cucullata and the hybrids $D$. cucullata $\times$ galeata, $D$. cucullata $\times$ hyalina and D. galeata $\times$ hyalina (Wolf and Mort 1986, Spaak 1995a). The fish fauna of the Plußsee consists of 13 species; roach (Rutilus rutilus) and perch (Perca fluviatilis) comprise about $70 \%$ of the standing stock (Krambeck et al. 1994).

Samples were taken by vertical hauls with a plankton net on 5 September 1995, and were transported to the laboratory. Adult females with parthenogenetic eggs were randomly taken from the lake sample and reared individually in the laboratory. All animals were assayed at three allozyme loci. The enzymes and their EC (Enzyme Commission) codes were: glutamate oxaloacetate transaminase (Got, EC 2.6.1.1), phosphoglucomutase (Pgm, EC 5.4.2.2) and phosphoglucose isomerase (Pgi, EC 5.3.1.9). All electrophoreses were carried out on Titan III ${ }^{\circledR}$ cellulose acetate plates following standard methods (Hebert and Beaton 1989). In this way, we obtained 95 isolates with known genotypes. Got was used to identify the taxa (Wolf and Mort 1986). For each taxon, we chose, if possible, four clones with a different multi-locus genotype. Due to low genetic variation we could only find one and two multi-locus genotypes for D. cucullata $\times$ hyalina and D. galeata $\times$ hyalina, respectively (Table 1). Hence, although we used different isolates from the lake for these last two taxa, we are not sure that they are indeed different clones.

\section{Life-table experiment}

For each clone used in the experiment, neonates were collected from the stock cultures, and 6-10 individuals

Table 1. Multi-locus genotypes of the 20 clones used in the life-history experiment. The clone numbers are used in all figures for identification.

\begin{tabular}{lllll}
\hline Taxon & Clone & Got & Pgi & Pgm \\
\hline D. galeata & 1 & FF & MF & FF \\
& 2 & FF & MF & MM \\
& 3 & FF & MM & FF \\
D. cuc. $\times$ gal. & 4 & FF & FF & SF \\
& 1 & S-F & MM & SF \\
& 2 & S-F & S-M & SF \\
D. cucullata & 1 & S-F & SM & MM \\
& 2 & S-F & SF & MF \\
& 3 & S-S- & SS & MM \\
& 4 & S-S- & S-S & SF \\
D. cuc. $\times$ hyl. & 1 & S-S- & S-M & SS \\
& 2 & S-S & SM & MF \\
& 3 & S-S & SM & MF+ \\
& 4 & S-S & SM & MF+ + \\
D. gal. $\times$ hyl. & 1 & S-S & SM & MF+ \\
& 2 & SF & MM & MF \\
& 3 & SF & MM & MF+ \\
& 4 & SF & MM & MF \\
& & SF & MM & MF \\
\hline
\end{tabular}

were placed together in $200-\mathrm{ml}$ jars. These individuals, the grandmothers of the experimental animals, were kept at $20^{\circ} \mathrm{C}$, in a long-day photoperiod (16:8 L:D). The green alga Scenedesmus obliquus served as food, with a concentration of $0.7 \mathrm{mg} \mathrm{C} 1^{-1}$ which is above the incipient limiting level (Boersma and Vijverberg 1994b). The algae were cultured in 3-1 chemostats on a Chu-12 medium (Lampert 1976), and harvested daily from the overflow. Daphnids were cultured in $0.45-\mu \mathrm{m}$ filtered lake water taken from the nearby Schöhsee. The cultures were refreshed daily. First-brood neonates of these animals were isolated to serve as mothers for the experimental animals, and subjected to the same conditions as their mothers. The neonates from the first brood of these animals were used in the life-table experiment, and maintained under the same food and temperature conditions as the maternal and grandmaternal animals.

The experimental vessels $(200 \mathrm{ml})$ were stocked with one neonate per vessel, and subjected to a fish water/ no-fish water treatment using five replicates per clone, resulting in 5 (taxa) $\times 4$ (clones) $\times 2$ (treatment $) \times 5$ (replicates) $=200$ experimental vessels. Some of the animals died during the course of the experiment due to handling. Fish water was obtained from a 30-1 aquarium which contained three fish (Leuciscus idus L.) of approximately $5 \mathrm{~cm}$ in length. Every day two-thirds of the water was collected from this aquarium, filtered through a $0.45-\mu \mathrm{m}$ filter, and aerated for $10 \mathrm{~min}$ to remove small air bubbles. The aquarium was refilled with unfiltered Schöhsee water. The fish were fed frozen chironomid larvae in a separate aquarium, where the faeces were deposited as well.

For three successive adult instars we counted the number of eggs in the brood pouch and the released newborns. For every adult instar, we measured the body length from the topside of the eye to the base of the spine of the mother and two of her produced newborns.

\section{Predation model}

The life-history traits calculated above are most likely important components of fitness, but do not represent fitness completely. Even the intrinsic rate of increase, $r$, which is often used as a synonym for fitness (e.g. Roff 1992), is not a proper fitness estimate when established from laboratory experiments, as it is calculated without natural mortality. Therefore, we computed rates of population increase $(r)$ of the animals in the life-history experiment under two different mortality schemes. If the reaction to fish kairomones of the different Daphnia taxa is adaptive then animals grown in medium with fish factor should have highest values of $r$ under a fish-type mortality regime compared to the controls cultured in water without the fish factor when predator 


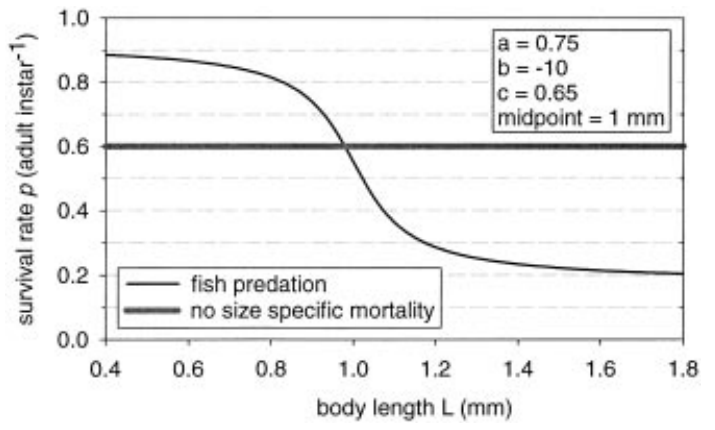

Fig. 1. Survival rate $p$ as a function of body length $L(\mathrm{~mm})$ The arctangent function (see text) models fish predation. The non-selective survival $(0.6)$, which was used to calculate $r$, is also indicated in the figure.

mortality is not included. We used the arctangent survival-rate model of Taylor and Gabriel (1992). In this model the survival rate, $p$, over an instar of duration $D$ days is a function of length $L$ in millimetres:

$p=\left\{a / \pi \arctan \left[b\left(L-L_{\text {mid }}\right)\right]+c\right\}^{\left(D / D_{-} A D\right)}$,

where $D_{-} A D$ is adult instar duration and $L_{\text {mid }}$ is the midpoint of the threshold region. With this threshold model, survival rates for Daphnia shift from high to low over a narrow size range. Two mortality regimes were tested (Fig. 1), one mimicking fish selectivity, with a decrease in survival probability with length $(a=0.75$; $b=-10 ; c=0.65$; midpoint $=1 \mathrm{~mm}$ ), and one mimicking no size-selective mortality with $p=0.6$. These survival rates were incorporated in the Euler-Lotka equation, and $r$ values for all clones under both predation regimes were computed. Taylor and Gabriel (1992) used their model for Daphnia magna, and presented their parameters $(a=0.5 ; b=-10 ; c=0.65$ for fish predation) without any background information. Parameter $a$ determines the minimal and maximal survival rate of the function. The minus sign of the $b$ parameter determines higher $p$ values at smaller body length. An increase in $b$ gives a steeper curve. The $c$ parameter determines the $p$ value at the midpoint $L_{\text {mid }}$. The midpoint is the length at which the shape of the curve inverts. Hence, we chose the parameters according to our knowledge about the predation risk by planktivorous fish for the Daphnia galeata complex (Boersma et al. 1996, Spaak and Hoekstra 1997). From our previous work, it is known that more than $60 \%$ of the Daphnias larger than $1.6 \mathrm{~mm}$ die per day due to fish predation. But also from the juveniles a certain percentage does not survive, estimated as $10 \%$ during the duration comparable to the duration of an adult instar. Values of $a=0.75$ and $c=0.55$ meet this criterion. For $b$ we used the same value as Taylor and Gabriel (1992). Boersma et al. (1996) showed that $0^{+}$fish prefers Daphnia larger then $1 \mathrm{~mm}$, therefore this value was used for $L_{\text {mid }}$. The non-selective survival rate was so chosen that the mean $r$ for all individual clones was the same as with the size-selective survival rate.

The data were analysed using analysis of variance with the following independent variables: fish factor, taxon, and clone nested within taxon. Clone was used as a random factor. We investigated a set of size-related traits (e.g. size at maturity, newborn size) and reproductive traits (e.g. number of eggs in the first adult instar and age at maturity). The size-related traits were log-transformed to correct for possible erroneously significant interactions. The rate of increase $r$ was calculated twice. Once with the size-dependent survival rate as described above (fish predation) and once with a constant survival rate of 0.6 (non-selective predation). Differences between means were analysed with a Tukey HSD multiple comparisons test for unequal sample sizes.

To investigate if fish predation contributes to the coexistence of these five taxa we computed the relative fitness of the five taxa under the fish predation and the non-selective predation regime. The highest $r$ value under both conditions was set at 1 (in most cases for $D$. cucullata and D. galeata $\times$ hyalina, respectively). Under the assumption that $2 / 3$ of the growing season (June till September) a fish-type selective predation occurs and $1 / 3$ of the growing season (May and October) a non-selective predation type we calculated a geometric average. This measure of relative fitness was also used as a measure for the parameter analyses of the size-dependent survival model. From all four parameters we tested five different values keeping the others constant at the levels shown in Fig. 1. Since relative fitness is compared we used in all calculations a non-selective survival rate of 0.6 .

\section{Results}

\section{Life-history traits}

D. galeata was the largest species (average size at maturity $1.51 \mathrm{~mm}$ ), followed by $D$. galeata $\times$ hyalina, $(1.35 \mathrm{~mm})$. Next were the hybrids between $D$. cucullata and $D$ galeata $(1.04 \mathrm{~mm})$, and between $D$. cucullata and D. hyalina $(1.01 \mathrm{~mm})$. D. cucullata was the smallest $(0.78 \mathrm{~mm})$ taxon. Only the size difference between $D$. cucullata $\times$ hyalina and D. cucullata $\times$ galeata was nonsignificant (Fig. 2A). Size at maturity showed a significant reaction to the fish factor (Table 2). The three largest taxa reacted to the fish factor with a significantly smaller size at maturity (D. galeata, D. galeata $\times$ hyalina and D. cucullata $\times$ galeata; Tukey HSD, $P<$ $0.001)$, whereas the differences for the two other taxa (D. cucullata and D. cucullata $\times$ hyalina) were non-significant (Fig. 2A). This different reaction of the taxa to fish factor is expressed by a highly significant fish factor $\times$ taxon interaction (Table 2 ). 
A significant taxon effect was also found for newborn size (Table 2), with roughly the same size sequence as with size at maturity. Although the overall effect of fish factor, causing the production of smaller offspring, was significant, post hoc comparisons showed that this was caused by the reaction of only two taxa (D. cucullata $\times$ galeata and D. galeata $\times$ hyalina) (Tukey HSD, $P<$ 0.002 ). These differences in offspring size were a direct result of the differences in the size at maturity of the mothers between the fish and no-fish treatment. Including size at maturity as a covariable in the analysis caused the effect of fish factor to become nonsignificant.

For both size-related traits, highly significant clone effects were found (Table 2). All but one of the reaction norms of the several clones for size at maturity were negative (Fig. 2A), i.e. the animals were smaller with fish factor present. Although the majority of the clones also had negative reaction norms for newborn size (15 out of 20), we observed more variation (Fig. 2B). Where the reaction norms of $D$. cucullata and D. cucullata $\times$ hyalina lie close to each other, a convergence is observed for $D$. cucullata $\times$ galeata, from a wide range under non-fish conditions to a very narrow range when fish kairomones were present.

The number of eggs in the first brood and age at maturity showed opposite patterns. Age at maturity showed no taxon effect but strong fish factor and clone effects (Fig. 3), whereas first brood size showed only a taxon effect (Table 2, Fig. 4). D. galeata and D. galeata $\times$ hyalina produced the highest numbers of eggs (7.4 and 7.2, respectively), which were not significantly different. The difference between $D$. cucullata $\times$ galeata and $D$. cucullata $\times$ hyalina was also non-significant $(4.0$ and 4.3). D. cucullata produced the smallest brood, 2.7 on average (Fig. 4).

In contrast to the size of the first brood, age at maturity showed no taxon effect but strong fish factor and clone effects (Table 2, Fig. 3). This fish factor effect was different for several taxa (Table 2), and was caused by a very strong reaction of $D$. cucullata $\times$ galeata $\left(F_{1,150}=29.59, P<0.0001\right)$ and a weaker reaction of $D$. cucullata $\left(F_{1,150}=4.46, P<0.04\right)$, while neither the other three taxa nor clones within these taxa showed a

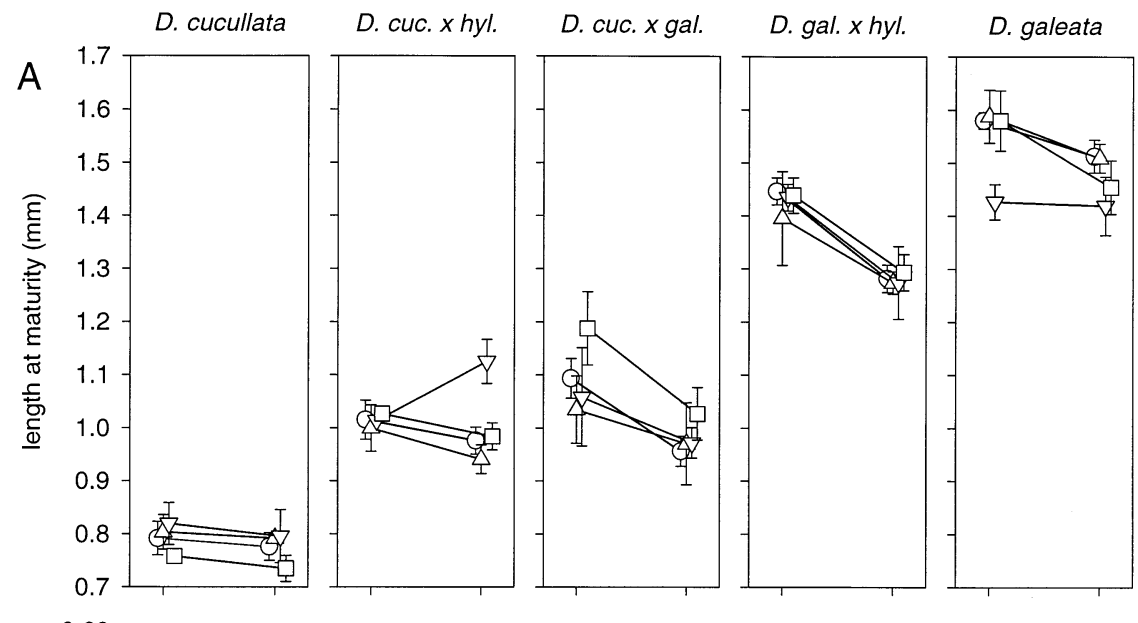

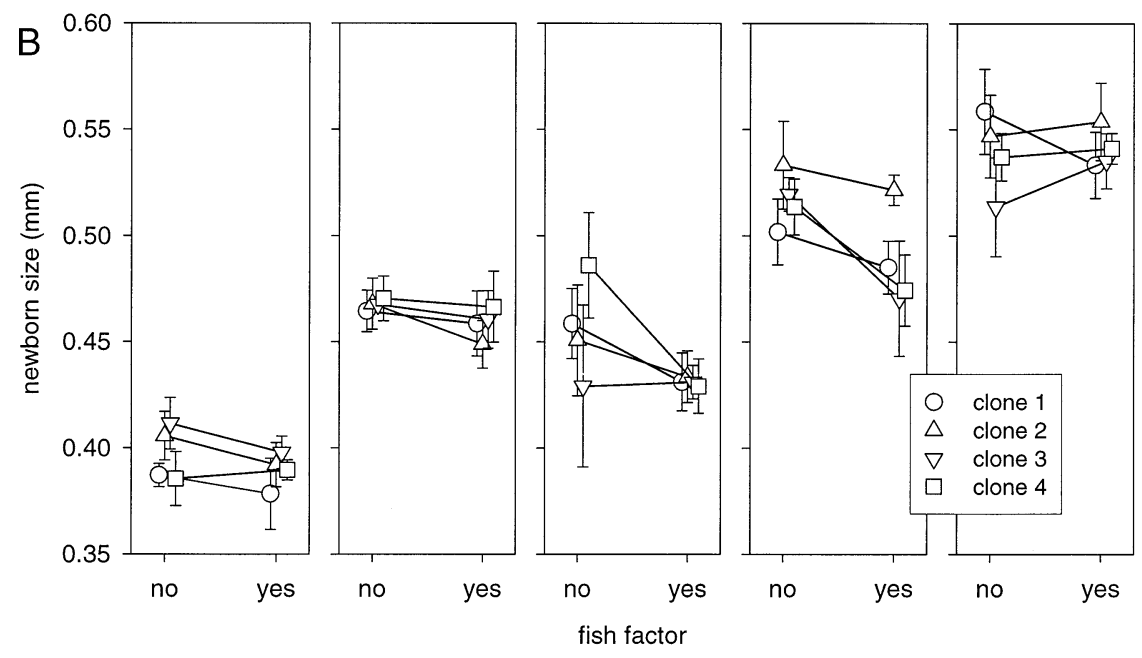

Fig. 2. Interaction plots of mean lengths at maturity and newborn sizes (of the first brood) with $95 \%$ confidence limits against the presence of fish factor for five Daphnia taxa. For each taxon the reaction of four clones are plotted. Clone numbers refer to Table 1. 


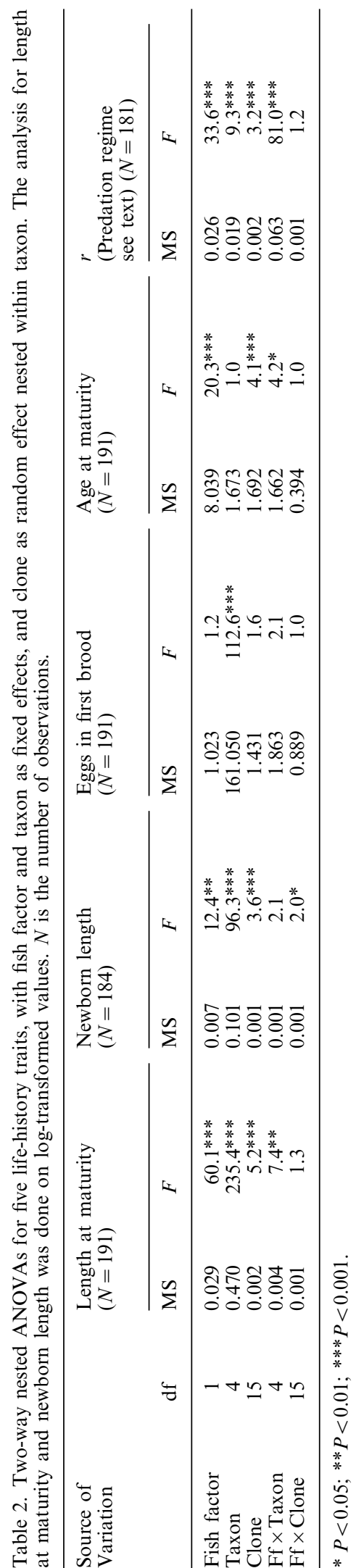

OIKOS 89:1 (2000) significant reaction to fish factor (Fig. 3). Although no taxon effect was found, we found large differences between the taxa with respect to the clonal variation for age at maturity (Fig. 3).

\section{Fitness comparisons}

In the case of non-selective mortality, three groups could be identified with non- significantly different $r$ values (i.e. D. galeata and $D$. galeata $\times$ hyalina; $D$. cucullata $\times$ hyalina and D. cucullata $\times$ galeata and $D$. cucullata) (Fig. 5). None of the interactions were found to be significant, and only the main effects taxon and clone were significant. However, for the $r$-values calculated with the size specific survival $\left(r_{\text {fish }}\right)$ a highly significant effect of fish factor was found $\left(F_{1,15}=41.8\right.$, $P<0.001)$. D. cucullata and D. cucullata $\times$ galeata showed a significant increase of $r_{\text {fish }}$ in reaction to the presence of kairomones $\left(F_{1,141}=6.9, P<0.01 ; F_{1,141}=\right.$ 49.4, $P<0.001)$, due to the combined effect of a strong reaction of age at maturity to fish factor and the decrease in body size $(D$. cucullata $\times$ galeata $)$ cq the small body size of $D$. cucullata. However, from the 20 clonal reaction norms for $r_{\text {fish }} 18$ were positive. Finally we compared the $r_{\text {fish }}$ of animals cultured under fish conditions with the $r_{\text {non-selective }}$ of the animals cultured under non-fish conditions in an ANOVA (Table 2) to test if taxa really differ under these conditions. We found a very strong fish factor $\times$ taxon interaction (Table 2), showing that the differences in $r$ between the taxa lead to fitness differences under specific mortality regimes.

For all traits, we computed a Spearman rank correlation coefficient between the size at maturity without fish and the relative change in all the traits measured when exposed to fish chemicals, as we hypothesised that the larger clones should show the largest changes. Only for size at maturity, this correlation was significant $(r=$ $0.53, P=0.015, n=20)$. For the 12 other traits we found no significant positive correlations. In fact, for $r_{\text {fish }}$ we observed a negative correlation, indicating that the smaller clones showed the strongest response (Fig. 5). This correlation was, however, only marginally significant $(r=-0.45, P=0.049, n=20)$.

\section{Parameter analysis}

To investigate how sensitive our results are to changes in the parameters of the size-dependent survival model, we calculated relative fitness values for different parameter settings. All four parameters of the size-dependent survival model were varied around the parameter set we chose for our analysis $(a=0.75, b=-10$, $c=0.55, L_{\mathrm{mid}}=100$; Fig. 1). The right panels of Fig. 6 show how the survival curve changes, the left panels show the changes in relative fitness. The relative fitness 

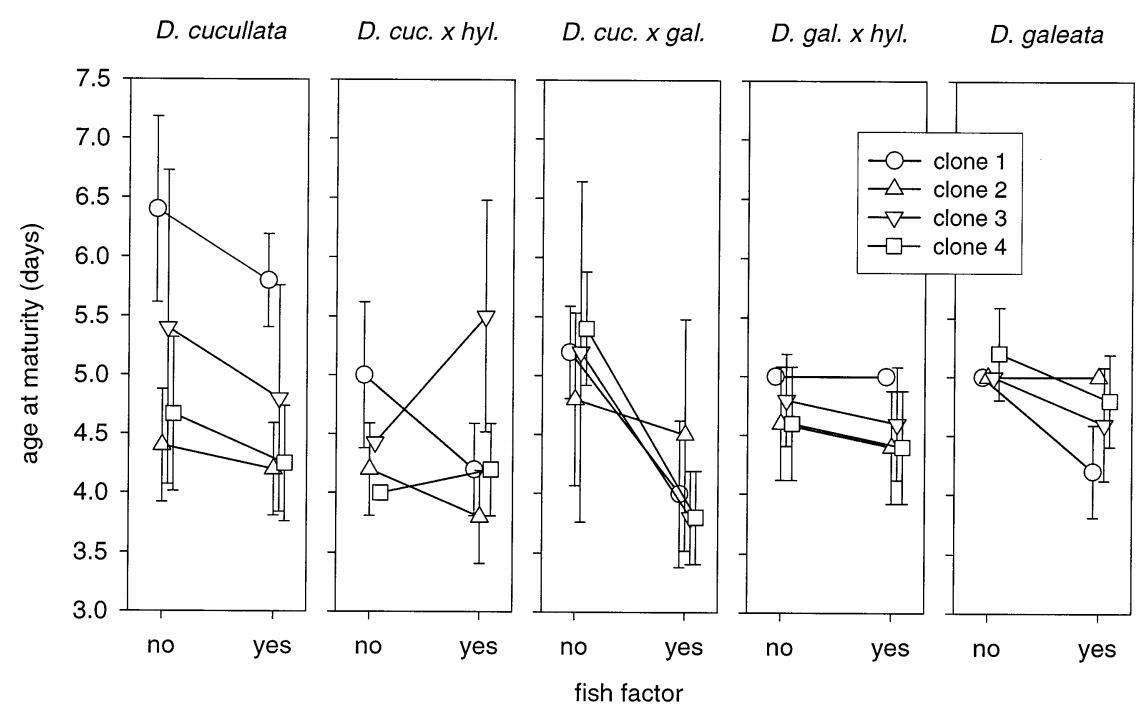
Fig. 3. Interaction plots of ages at maturity as in Fig. 2.

curve for the five taxa results from the geometric average of the $r_{\text {fish }}$ and the $r_{\text {non-selective }}$ values (Fig. 7).

The largest impact on the relative fitness curve have changes in the $c$ and $L_{\text {mid }}$ parameter. Changes in $c$ move the whole curve down, resulting in large changes, especially for the larger taxa $D$. galeata $\times$ hyalina and $D$. galeata when $c \leq 0.5$ (Fig. 6C). The smaller taxa $D$. cucullata $\times$ hyalina and D. cucullata $\times$ galeata react the strongest at the reduction of $L_{\text {mid }}$ below $1 \mathrm{~mm}$ (Fig. $6 \mathrm{D}$ ). The model is quite robust for changes of $b$ (Fig. 6B); only a value of $b=-2$ changes the pattern of the relative fitness curves completely. Changes in $a$ do not change the shape of the fitness curve, but the differences between the two large taxa and the three smaller taxa become more pronounced at higher values of $a$ (Fig. 6A).

\section{Discussion}

Several studies have shown that daphnids can change their life histories in the presence of fish kairomones (Brett 1992, Stibor 1992, Macháček 1995, Tollrian 1995). A few studies exist describing clonal differences in this reaction (Weider and Pijanowska 1993, Reede and Ringelberg 1995), but these studies were carried out with a limited number of clones only. Using multiple clones from multiple taxa, we were able to generalise the results from these previous studies, i.e. animals mature at an earlier age, and at a smaller size. However, our study also shows that clones from five taxa of the D. galeata complex, which co-occur in Plußsee, not only differ considerably in their life-history characters, but also in the amplitude with which these traits change in reaction to the presence of fish chemicals.

The fact that we find differences in life-history characteristics between clones from different taxa is not

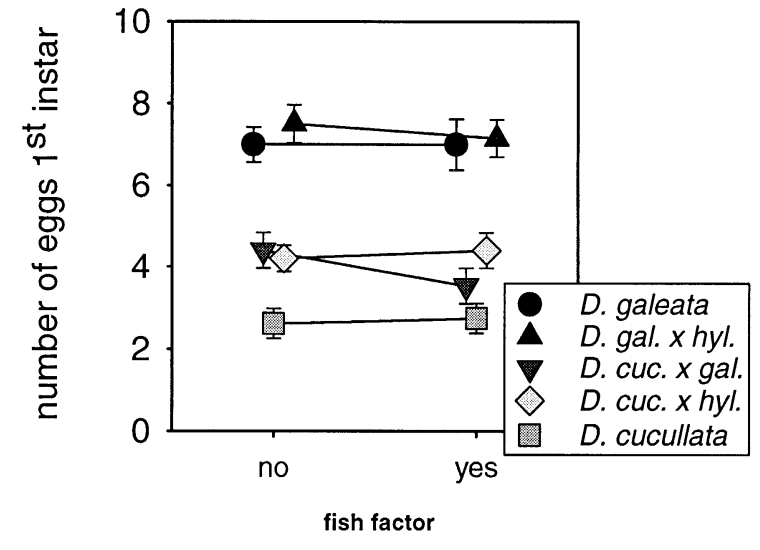

Fig. 4. Interaction plots of means and $95 \%$ confidence limits of the size of the first brood against fish factor, plotted for the five different taxa. 
Fig. 5. Interaction plots of means and $95 \%$ confidence limits of the rate of increase, $r$, calculated with two different survival models. The 'fish' model used the size-dependent arctangent survival model, whereas for the calculation of the 'non-selective $r$ ' a constant survival rate of 0.6 was used.

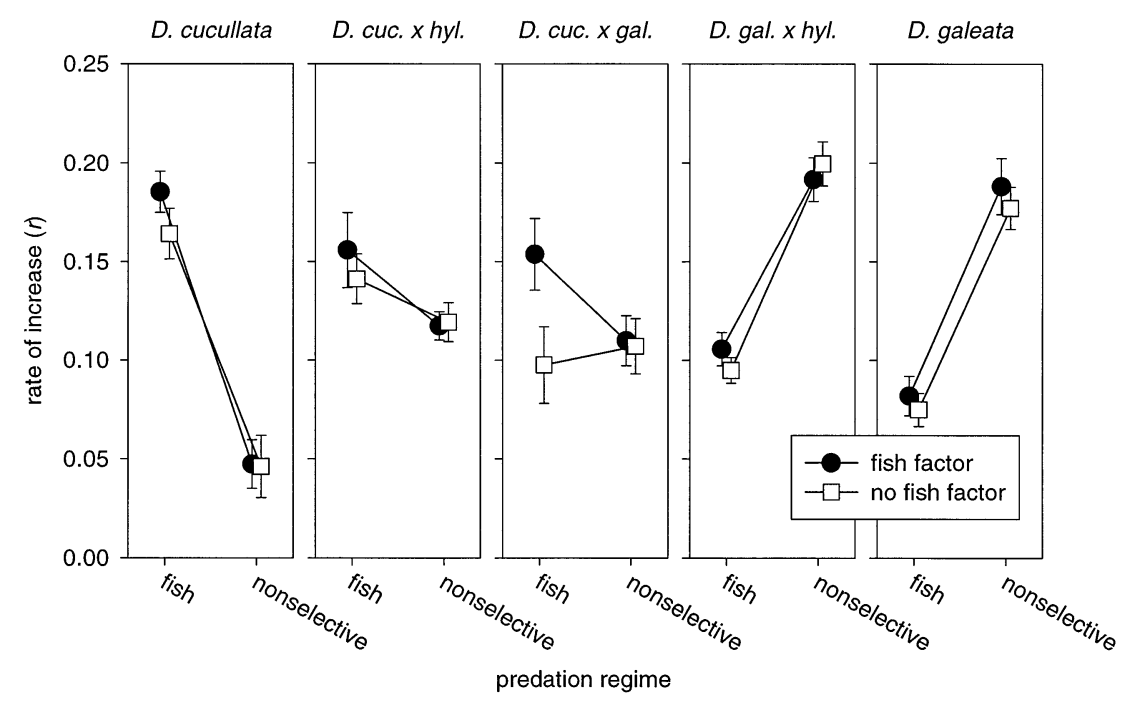

(e.g. Boersma et al. 1996). Therefore, the selection pressure on body size of daphnids can be expected to be greater for larger taxa, and their reaction to fish chemicals more severe. Indeed, we found a significant correlation between the percentage of reduction of size at maturity under conditions with fish chemicals present and the initial size at maturity of the clones.

In contrast to the changes in size at maturity caused by the presence of fish kairomones, which were greater for the larger taxa, we found no such differences for age at maturity. In fact, the changes in age at maturity seem more dramatic in the smaller taxa (Fig. 3), and as a result, as egg numbers hardly changed, also the values for the rate of increase, $r$, were more plastic for the smaller taxa (data not shown). Values for $r$ are often used as a measure of fitness (Stearns 1992). It is difficult to conceive, however, why animals would increase their fitness when exposed to chemicals produced by fish, as this would mean that their fitness is sub-optimal when no fish are present. This apparent paradox can be explained by the way $r$ is computed. Laboratoryderived values for $r$ do not incorporate mortality under field conditions. The presence of fish will mostly mean that mortality is higher, especially for the larger individuals. Hence, under natural conditions it will be most unlikely to see an increase in fitness as a result of fish predation. Size-selective predation by fish can also explain why the smaller taxa can coexist with the larger ones in this lake although the number of newborns for these smaller taxa is considerably smaller than for the larger taxa. Although the larger taxa show a stronger reduction in size in the presence of fish, the individuals of these taxa are still larger than the individuals of the smaller taxa. Hence, they will still be more vulnerable to predation than individuals of the smaller taxa. The $r_{\text {fish }}$ values we computed take these differences in size into account. When a non-selective predation model was used, $r$ values were the highest for the large taxa, which produce the most offspring (D. galeata and $D$. galeata $\times$ hyalina $)($ Fig. 5). But with the size-specific predation model $r$ was the lowest for these two taxa. Although $r_{\text {fish }}$ of $D$. cucullata $\times$ galeata clones cultured without fish factor was not significantly different from the two largest taxa, a strong significant reaction on fish factor was found. Also for D. cucullata this reaction was significant, leading to a significant negative correlation (computed over all taxa) between the size at maturity (without fish factor) and the reaction to fish factor for this trait.

Size and age at maturity in daphnids are usually positively correlated (Ebert 1991), i.e. animals maturing at a later age are larger. It is therefore possible that the observed changes in age at maturity in the presence of fish chemicals are a result of pleiotropic effects as a result of selection on size at maturity rather than direct selection on early reproduction. The fact that Boersma et al. (1998) found a significant genetic correlation between these traits in Daphnia magna indeed suggests such a linkage. The increase in $r$ would then be a side effect of such selection on smaller size at maturity. It could be argued that animals should also stay smaller when fish chemicals are not present, as the measured fitness is higher for smaller animals. However, a smaller size also means smaller newborns (Fig. 2B). Smaller newborns have a lower resistance to starvation (Threlkeld 1976), and hence the competitive abilities of these animals would be lower (e.g. Gliwicz and Lampert 1990). Furthermore, smaller neonates are more vulnerable to invertebrate predators, which have a higher abundance when fish are absent.

Our comparisons of the relative fitness (Fig. 7) show that the average mean relative fitness over the whole growing season is almost the same for these five taxa. This supports our hypothesis that fish predation might 

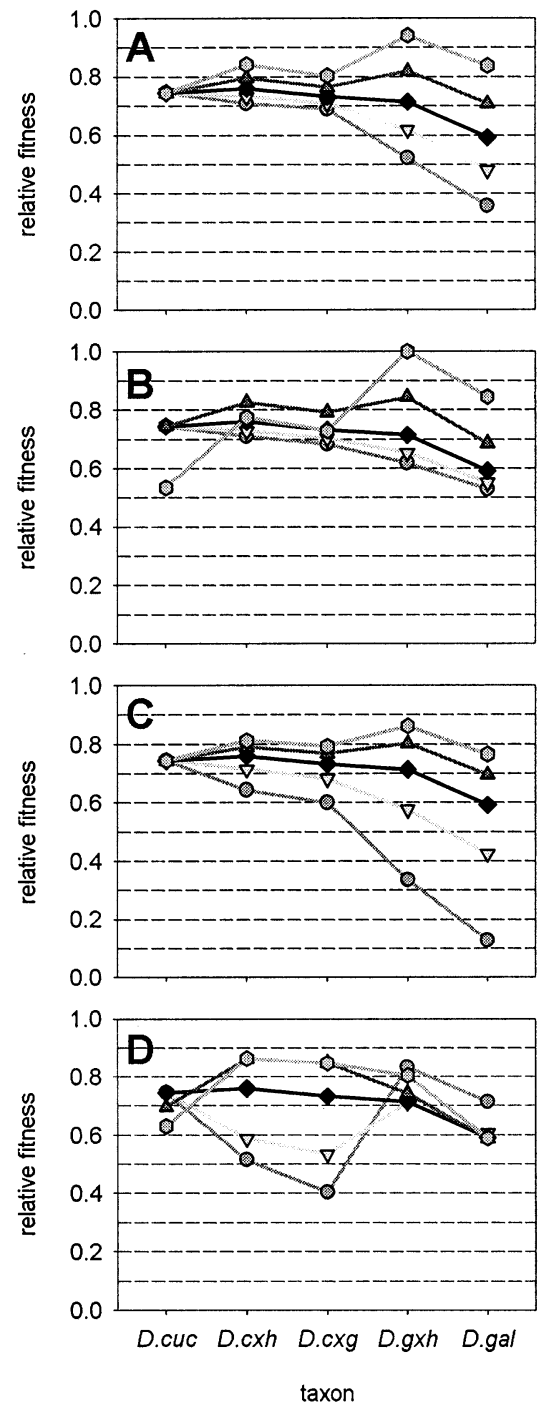
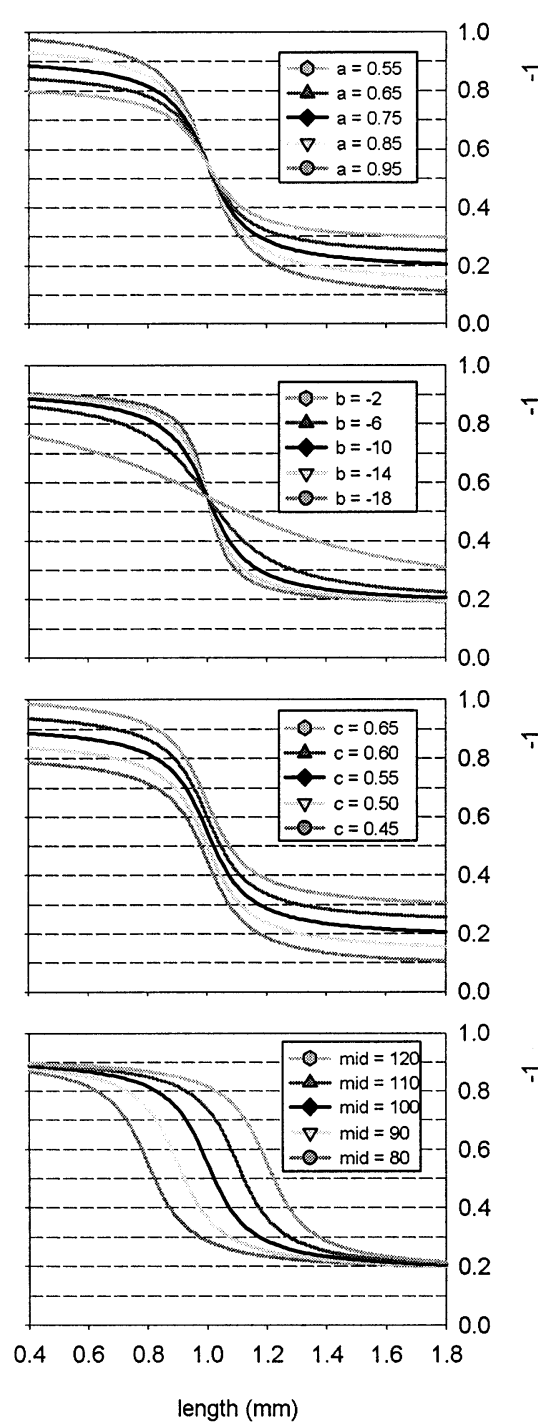

Fig. 6. Influence of parameter changes of the survival model (see text) on the relative fitness of the five Daphnia taxa. The relative fitness lines (left panels) are the geometric average between the $r_{\text {fish }}(2 / 3)$ and $r_{\text {non-selective }}(1 / 3)$. One parameter was varied at a time keeping the other three constant. Therefore the black survival curves, and the black line $(\diamond)$ with relative fitnesses, are the same in all four graphs, and can be used as reference. be a factor that facilitates the co-occurrence of parental and hybrid taxa. The parameter analysis of the size-dependent survival model shows, furthermore, that our results are quite robust. As expected, changes in $L_{\text {mid }}$ and $c$ influence relative fitness the most, but some variation around the parameter set we chose changes the relative position of the relative fitness curve, but not its shape (Fig. 6). The fact that changing the parameters of the survival has the largest effect on the larger taxa has to do with the fact that we used always the same survival rate for the calculation of $r_{\text {non-selective. }}$ Because $r_{\text {fish }}$ has almost always the highest value for the smallest taxon (D. cucullata), and therefore the relative fitness of this taxon under fish predation is 1 by definition, we see no or little variations for these taxa when the parameters of the survival model are changed.

The present study is the first to our knowledge comparing the changes in parental and hybrid taxa caused by the presence of fish chemicals. We observed a relationship between the initial size of the clones, and the percentage decrease in size at maturity, a phenomenon which is probably adaptive since these taxa face the highest predation pressures. Incorporating sizeselective mortality in the computations of $r$ showed that the observed life histories under fish conditions could increase the relative fitness of the smaller taxa significantly. From this we conclude that the presence of fish is at least one of the factors causing the co-occurrence of several Daphnia taxa in Plußsee.

Acknowledgements - During the experimental phase of this study PS and MB were supported by Max Planck Society fellowships. JV is a fellow of the Flemish Institute for the Promotion of Scientific-Technological Research in Industry (IWT); he was supported by a travelling grant from the Max Planck Society. PS is supported by a grant from the Swiss Federal Office for Education and Science. MB is supported by 


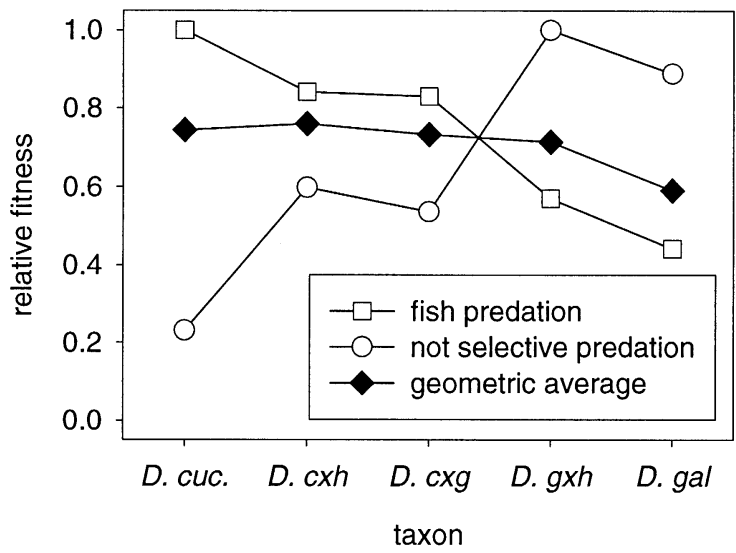

Fig. 7. Summary graph of the relative fitness for the fish and no selective survival models. The highest value was set at $1(D$. cucullata and D. galeata $\times$ hyalina, respectively).). The "whole growing season' values were calculated as geometric means under the assumption that during $2 / 3$ of the growing season fish-type selective predation occurs and during $1 / 3$ a non-selective predation type (see text).

contract ENV4-CT97-0402 within the framework of the European Commission's Environment and Climate Programme and is part of the project network WatER (Wetland and Aquatic Ecosystem Research). We thank Larry Weider, Winfried Lampert, James Ward and Chris Robinson for their support and comments on different versions of the manuscript.

\section{References}

Anderson, E. and Stebbins, G. L. 1954. Hybridization as an evolutionary stimulus. - Evolution 8: 378-388.

Arnold, M. L. 1997. Natural hybridization and evolution. Oxford Univ. Press, Oxford.

Barton, N. H. and Hewitt, G. M. 1985. Analysis of hybrid zones. - Annu. Rev. Ecol. Syst. 16: 113-148.

Barton, N. H. and Hewitt, G. M. 1989. Adaptation, speciation and hybrid zones. - Nature 341: 497-503.

Boersma, M. and Vijverberg, J. 1994a. Seasonal variations in the condition of two Daphnia species and their hybrid in a eutrophic lake: evidence for food limitation. - J. Plankton Res. 16: 1793-1809.

Boersma, M. and Vijverberg, J. 1994b. Resource depression in Daphnia galeata, Daphnia cucullata and their interspecific hybrid: life history consequences. - J. Plankton Res. 16: $1741-1758$.

Boersma, M., Van Tongeren, O. F. R. and Mooij, W. M. 1996. Seasonal patterns in the mortality of Daphnia species in a shallow lake. - Can. J. Fish. Aquat. Sci. 53: 18-28.

Boersma, M., Spaak, P. and De Meester, L. 1998. Predatormediated plasticity in morphology, life history, and behavior of Daphnia: the uncoupling of responses. - Am. Nat. 152: 237-248.

Brett, M. T. 1992. Chaoborus and fish-mediated influences on Daphnia longispina population structure, dynamics and life history strategies. - Oecologia 89: 69-77.

Darwin, C. 1859. On the origin of species by means of natural selection, or the preservation of favoured races in the struggle for life. - John Murray, London.

Ebert, D. 1991. The effect of size at birth, maturation threshold and genetic differences on the life-history of Daphnia magna. - Oecologia 86: 243-250.

Endler, J. A. 1977. Geographic variation, speciation, and clines. - Princeton Univ. Press, Princeton, NJ.
Fiksen, O. 1997. Allocation patterns and diel vertical migration: modeling the optimal Daphnia. - Ecology 78: 14461456.

Gliwicz, Z. M. and Lampert, W. 1990. Food thresholds in Daphnia species in the absence and presence of blue-green filaments. - Ecology 71: 691-702.

Grant, P. R. 1993. Hybridization of Darwin finches on Isla Daphne Major, Galápagos. - Philos. Trans. R. Soc. Lond. B 340: 127-139.

Grant, P. R. and Grant, B. R. 1992. Hybridization of bird species. - Science 256: 193-197.

Grant, V. 1981. Plant speciation. - Columbia Univ. Press, New York.

Hann, B. J. and Hebert, P. D. N. 1982. Re-interpretation of genetic variation in Simocephalus (Cladocera, Daphniidae). - Genetics 102: 101-107.

Harrison, R. G. Ed. 1993. Hybrid zones and the evolutionary process. - Oxford Univ. Press, Oxford.

Hebert, P. D. N. and Payne, W. J. 1985. Genetic variation in populations of hermaphroditic flatworms Mesostoma lingna (Turbellaria: Rhabdocoela). - Biol. Bull. 169: 143151

Hebert, P. D. N. and Beaton, M. J. 1989. Methodologies for allozyme analysis using cellulose acetate electrophoresis. Helena laboratories Beaumont.

Kerfoot, W. C. and Sih, A. (eds). 1987. Predation: direct and indirect impacts on aquatic communities. Univ. Press of New England, Hanover, NH.

Krambeck, H., Albrecht, D., Hickel, B. et al. 1994. Limnology of the Plußsee. - In: Overbeck, J. and Chróst, R. (eds), Microbial ecology of Lake Plußsee. Springer-Verlag, New York, pp. 1-23.

Lampert, W. 1976. A directly coupled, artificial two-step food chain for long-term experiments with filter-feeders at constant food concentrations. - Mar. Biol. 37: 349-355.

Larsson, P. and Dodson, S. 1993. Invited review - chemical communication in planktonic animals. - Arch. Hydrobiol. 129: $129-155$

Lieder, U. 1987. The possible origin of Daphnia cucullata procurva Poppe 1887 in the lakes of the Pomerian Lakeland by hybridization in the past. - Hydrobiologia 145: $195-200$.

Lynch, M. 1980. The evolution of cladoceran life histories. Q. Rev. Biol. 55: 23-42.

Macháček, J. 1995. Inducibility of life history changes by fish kairomone in various developmental stages of Daphnia. J. Plankton Res. 17: 1513-1520.

Mayr, E. 1942. Systematics and the origin of species. Columbia Univ. Press, New York.

Mayr, E. 1963. Animal species and evolution. - Belknap Press of Harvard Univ. Press, Cambridge, MA.

Moore, W. S. 1977. An evaluation of narrow hybrid zones in vertebrates. - Q. Rev. Biol. 52: 263-277.

Reede, T. 1995. Life history shifts in response to different levels of fish kairomones in Daphnia. - J. Plankton Res. 17: $1661-1667$

Reede, T. and Ringelberg, J. 1995. The influence of a fish exudate on two clones of the hybrid Daphnia galeata $\times$ hyalina. - Hydrobiologia 307: 207-212.

Roff, D. A. 1992. The evolution of life histories. - Chapman \& Hall, New York.

Schwenk, K. 1997. Evolutionary genetics of Daphnia species complexes-hybridism in syntopy. - PhD thesis, Univ. of Utrecht, Utrecht.

Schwenk, K. and Spaak, P. 1995. Evolutionary and ecological consequences of interspecific hybridization in cladocerans. - Experientia 51: 465-481.

Schwenk, K. and Spaak, P. 1997. Ecological and genetics of interspecific hybridization in Daphnia. - In: Streit, B., Städler, T. and Lively, C. M. (eds), Evolutionary ecology of freshwater animals. Birkhäuser, Basel, pp. 199-229.

Shan, R. K. and Frey, D. G. 1983. Pleuroxus denticulatus and P. procurvus (Crustacea: Chydoridae) in North America: distribution, experimental hybridization and the possibility of natural hybridization. - Can. J. Zool. 61: 1605-1617. 
Solignac, M. and Monnerot, M. 1986. Race formation, speciation, and introgression within Drosophila simulans, Drosophila mauritiana, and Drosophila sechellia inferred from mitochondrial DNA analysis. - Evolution 40: 531539.

Sommer, U., Gliwicz, Z. M., Lampert, W. and Duncan, A 1986. The PEG-model of seasonal succession of planktonic events in fresh waters. - Arch. Hydrobiol. 106: 433-471.

Spaak, P. 1995a. Sexual reproduction in Daphnia: interspecific differences in a hybrid species complex. - Oecologia 104: 501-507.

Spaak, P. 1995b. Cyclomorphosis as a factor explaining success of a Daphnia hybrid in Tjeukemeer. - Hydrobiologia 307: 283-289.

Spaak, P. 1996. Temporal changes in the genetic structure of the Daphnia species complex in Tjeukemeer, with evidence for backcrossing. - Heredity 76: 539-548.

Spaak, P. and Hoekstra, J. R. 1995. Life history variation and the coexistence of a Daphnia hybrid with its parental species. - Ecology 76: 553-564.

Spaak, P. and Hoekstra, J. R. 1997. Fish predation on a Daphnia hybrid species complex: A factor explaining species coexistence? - Limnol. Oceanogr. 42: 753-762.

Stace, C. A. 1987. Hybridization and the plant species. - In: Krystyna, M. and Urbanska, K. M. (eds), Differentiation patterns in higher plants. Academic Press, London, pp. $115-127$.

Stearns, S. C. 1992. The evolution of life histories. - Oxford Univ. Press, Oxford.
Stibor, H. 1992. Predator induced life-history shifts in a freshwater cladoceran. - Oecologia 92: 162-165.

Taylor, B. E. and Gabriel, W. 1992. To grow or not to grow-optimal resource allocation for Daphnia. - Am. Nat. 139: $248-266$

Threlkeld, S. T. 1976. Starvation and the size structure of zooplankton communities. - Freshwat. Biol. 6: 489-496.

Tollrian, R. 1995. Predator-induced morphological defenses: costs, life history shifts, and maternal effects in Daphnia pulex. - Ecology 76: 1691-1705.

Weider, L. J. 1993. Niche breadth and life history variation in a hybrid Daphnia complex. - Ecology 74: 935-943.

Weider, L. J. and Wolf, H. G. 1991. Life-history variation in a hybrid species complex of Daphnia. - Oecologia 87: $506-513$.

Weider, L. J. and Stich, H. B. 1992. Spatial and temporal heterogeneity of Daphnia in Lake Constance; intra- and interspecific comparisons. - Limnol. Oceanogr. 37: 13271334.

Weider, L. J. and Pijanowska, J. 1993. Plasticity of Daphnia life histories in response to chemical cues from predators. - Oikos 67: 385-392.

Whitham, T. G., Morrow, P A and Potts, B. M. 1991 Conservation of hybrid plants. - Science 254: 779-780.

Wolf, H. G. and Mort, M. A. 1986. Interspecific hybridization underlies phenotypic variability in Daphnia populations. Oecologia 68: 507-511.

Zaret, T. M. 1980. Predation and freshwater communities. Yale Univ. Press, New Haven, CT. 